\title{
Spin Dynamics of a Canted Antiferromagnet in a Magnetic Field
}

\author{
R.S. Fishman \\ Condensed Matter Sciences Division, \\ Oak Ridge National Laboratory, Oak Ridge, TN 37831-6032
}

\begin{abstract}
The spin dynamics of a canted antiferromagnet with a quadratic spin-wave dispersion near $\mathbf{q}=0$ is shown to possess a unique signature. When the anisotropy gap is negligible, the spin-wave stiffness $D_{\mathrm{sw}}(\mathbf{q}, B)=\left(\omega_{\mathbf{q}}-B\right) / q^{2}$ depends on whether the limit of zero field or zero wavevector is taken first. Consequently, $D_{\mathrm{sw}}$ is a strong function of magnetic field at a fixed wavevector. Even in the presence of a sizeable anisotropy gap, the field dependence of the extrapolated $\mathbf{q}=0$ gap energy distinguishes a canted antiferromagnet from a phase-separated mixture containing both ferromagnetic and antiferromagnetic regions.
\end{abstract}


One of the greatest challenges in magnetism is to identify and characterize a canted antiferromagnet (CAF). Double quantum dots [1], cuprates [2], ruthenates [3], $\mathrm{RMn}_{2} \mathrm{Ge}_{2}$ compounds [4], Ho and Dy rare-earth borocarbides [5] and intermetallics [6], and lightlydoped manganites [7, 8, 9] are all believed to have a CAF phase. But in practice, it is extraordinarily difficult to distinguish a CAF from from a phase-separated mixture of a ferromagnet $(\mathrm{FM})$ and an antiferromagnet $(\mathrm{AF})$. This Letter demonstrates that a CAF with a quadratic spin-wave (SW) dispersion around $\mathbf{q}=0$ possesses a unique dynamical signature. In a magnetic field $B$, the SW stiffness $D_{\mathrm{sw}}(\mathbf{q}, B)=\left(\omega_{\mathbf{q}}-\omega_{0}\right) / q^{2}$ of a CAF with negligible anisotropy gap approaches different values depending on whether the limit of vanishing wavevector or field is taken first. Consequently, the SW stiffness for a fixed wavevector changes rapidly in small fields. Even when the anisotropy gap is sizeable, the field dependence of the extrapolated $\mathbf{q}=0$ energy gap still distinguishes a CAF from a phase-separated mixture containing FM regions. These results are used to demonstrate that the "FM" regions in $\operatorname{Pr}_{0.7} \mathrm{Ca}_{0.3} \mathrm{MnO}_{3}$ are actually canted.

The Hamiltonian of a system consisting of spins $\mathbf{S}_{i}$ at sites $i$ in a field along the $z$ direction can generally be written as $H=H^{(0)}-B \sum_{i} S_{i z}$ (set $2 \mu_{B}=1$ until it is needed). If inversion symmetry is unbroken [10] and the anisotropy gap is negligible, then the small $q \mathrm{SW}$ dispersion of a FM or CAF with net magnetization in the $z$ direction can be written as

$$
\omega_{\mathbf{q}}=\sqrt{\omega_{0}^{2}+2 \omega_{0} E_{0} q^{2}+D_{0}^{2} q^{4}}
$$

where $\omega_{0}=B$ is the energy gap and the wavevector $\mathbf{q}$ lies along one of the crystal axis. For simplicity, the lattice constant is set to 1.

In a FM, the transverse SW frequencies are obtained from the time dependence of $S_{i \pm}=$ $S_{i x} \pm i S_{i y}$. Since $\dot{S}_{i \pm}=i\left[H^{(0)}, S_{i \pm}\right] \mp i B S_{i \pm}$, the SW frequencies of a FM are simply shifted by $B$. So for a FM, $D_{0}=E_{0}$ and the small $q$ dispersion is given by $\omega_{\mathbf{q}}=B+D_{0} q^{2}$. For a CAF, the transverse components of the spin differ from one site to another and the equilibrium angles depend on field. Hence, the above argument fails. Because a magnetic field does not just shift the SW spectrum, it follows quite generally that $D_{0} \neq E_{0}$ in a CAF. This simple conclusion has some remarkable consequences. Notice that $D_{0}$ and $E_{0}$ are given by distinct limits of $D_{\mathrm{sw}}(\mathbf{q}, B): \quad D_{0}=\lim _{q \rightarrow 0} \lim _{B \rightarrow 0} D_{\mathrm{sw}}(\mathbf{q}, B)$ and $E_{0}=\lim _{B \rightarrow 0} \lim _{q \rightarrow 0} D_{\mathrm{sw}}(\mathbf{q}, B)$. When the limit of zero wavevector is taken first, $d \omega_{\mathbf{q}} / d B \rightarrow 1$ but when the limit of zero field is taken first, $d \omega_{\mathbf{q}} / d B \rightarrow E_{0} / D_{0} \neq 1$. At a fixed wavevector q, Eq.(11) implies that 


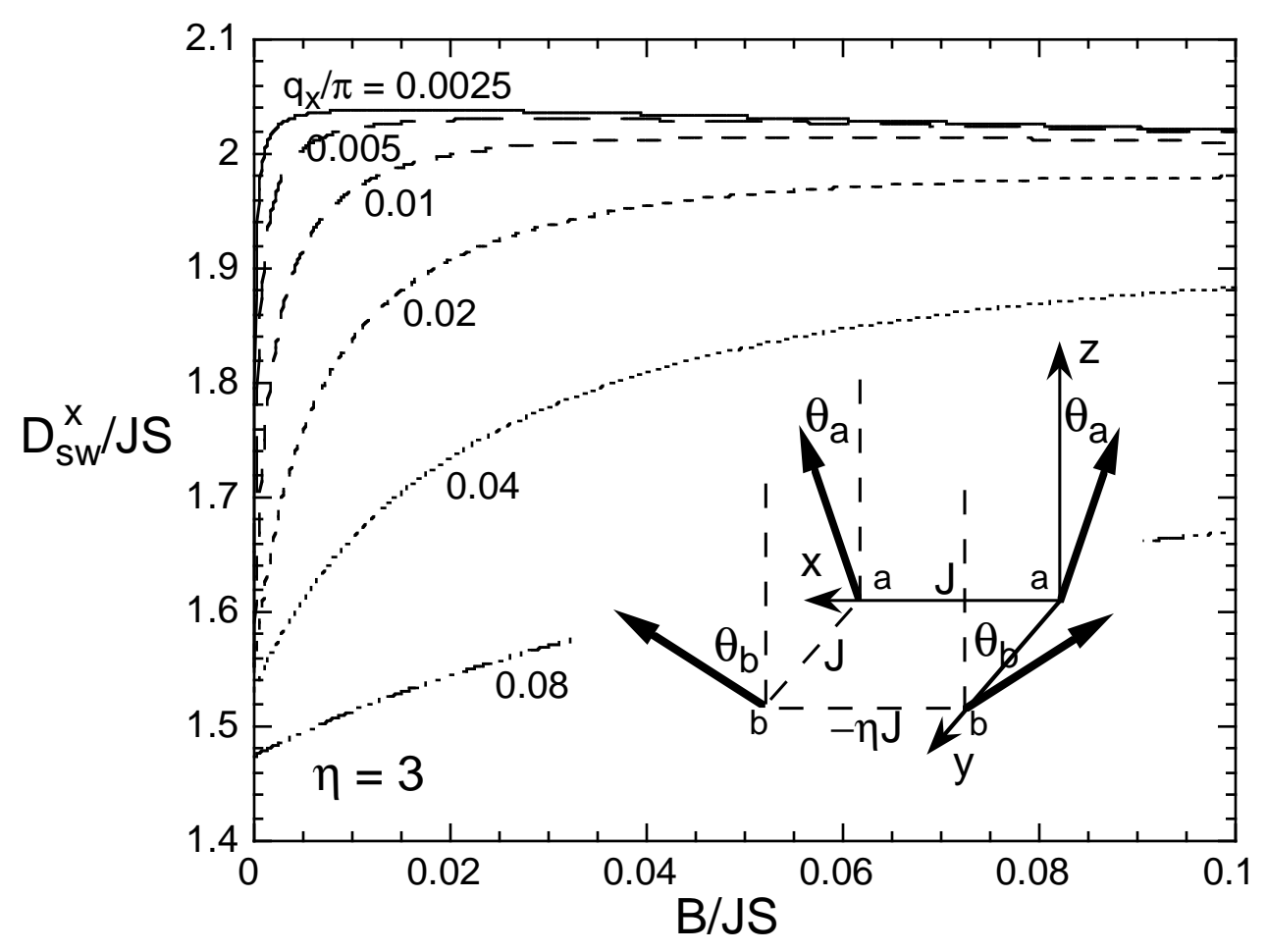

FIG. 1: The SW stiffness in the $x$ direction versus field for $q_{y}=0$ and various values of $q_{x} / \pi$ with $\eta=3$. Inset is a sketch of the GV model.

$D_{\mathrm{sw}}(\mathbf{q}, B)$ is a strong function of field when $B$ is in the neighborhood of $B^{\star} \equiv D_{0} q^{2}$. For fields much less than $B^{\star}, D_{\mathrm{sw}} \approx D_{0}$; for much larger fields, $D_{\mathrm{sw}} \approx E_{0}$.

To demonstrate these ideas, we consider one of the simplest models for a two-dimensional $\mathrm{CAF}$, which is the generalized Villain (GV) model [11, 12, 13] on a two-dimensional lattice with three-dimensional spins. As sketched in the inset to Fig.1, the spins on sublattice $a$ are FM coupled to each other and to the spins on sublattice $b$ with exchange constant $J>0$ while the spins on sublattice $b$ are AF coupled to each other with exchange constant $-\eta J$. The Hamiltonian of the GV model is $H=-\sum_{\langle i, j\rangle} J_{i j} \mathbf{S}_{i} \cdot \mathbf{S}_{j}-B \sum_{i} S_{i z}$, where the nearestneighbor exchange coupling $J_{i j}$ equals either $J$ or $-\eta J$. The CAF phase is stable when $\eta$ exceeds the critical value $\eta_{c}$, which is $1 / 3$ in zero field but increases as $B$ increases. Due to the different environments of the $a$ and $b$ sites, the angle $\theta_{b}$ at the $b$ sites is always larger than the angle $\theta_{a}$ at the $a$ sites.

The spin dynamics of the GV model is solved within the rotated reference frame for each spin, $\overline{\mathbf{S}}_{i}=\underline{U}_{i} \mathbf{S}_{i}$, where $\underline{U}_{i}$ is the unitary rotation matrix for site $i$. A Holstein-Primakoff expansion is performed within each rotated reference frame: $\bar{S}_{i z}=S-a_{i}^{\dagger} a_{i}, \bar{S}_{i+}=\sqrt{2 S} a_{i}$, 
and $\bar{S}_{i-}=\sqrt{2 S} a_{i}^{\dagger}$. Minimizing the ground-state energy $E=\langle H\rangle$ with respect to $\theta_{a}$ and $\theta_{b}$ yields the relations [13]

$$
\begin{gathered}
\sin 2 \theta_{a}+\sin \left(\theta_{a}-\theta_{b}\right)+\frac{B}{2 J S} \sin \theta_{a}=0, \\
-\eta \sin 2 \theta_{b}-\sin \left(\theta_{a}-\theta_{b}\right)+\frac{B}{2 J S} \sin \theta_{b}=0,
\end{gathered}
$$

where $B S$ is considered to be of the same order in $1 / S$ as $J S^{2}$. In zero field, it is easy to show that $\theta_{b}=3 \theta_{a}$ for all $\eta$.

After expanding $H=E+H_{1}+H_{2}+\ldots$ in powers of $1 / \sqrt{S}$, we find that the the firstorder term $H_{1}$ vanishes provided that the angles $\theta_{a}$ and $\theta_{b}$ satisfy Eqs.(2) and (3). In terms of the Fourier-transformed spin operators $a_{\mathbf{q}}^{(r)}$ and $a_{\mathbf{q}}^{(r) \dagger}$ on the $r=a$ or $b$ sublattice, the second-order term can be written as

$$
H_{2}=J S \sum_{\mathbf{q}, r, s}\left\{a_{\mathbf{q}}^{(r) \dagger} a_{\mathbf{q}}^{(s)} A_{\mathbf{q}}^{(r, s)}+\left(a_{-\mathbf{q}}^{(r)} a_{\mathbf{q}}^{(s)}+a_{-\mathbf{q}}^{(r) \dagger} a_{\mathbf{q}}^{(s) \dagger}\right) B_{\mathbf{q}}^{(r, s)}\right\},
$$

with coefficients $A_{\mathbf{q}}^{(r, s)}$ and $B_{\mathbf{q}}^{(r, s)}$ given elsewhere [14]. The Hamiltonian of Eq. (4) is easily diagonalized [14] using the method originally developed by Walker and Walstedt [15] for spin glasses. The resulting spin-wave frequencies in both the CAF and FM phases satisfy the condition $\omega_{0}=B$; in the CAF phase, $\omega_{\mathbf{Q}}=0$ where $\mathbf{Q}=(\pi, 0)$ is the AF Bragg vector. The results of this calculation agree with the SW frequencies numerically evaluated by Saslow and Erwin [16].

In the FM phase with $\eta<1 / 3$, the SW stiffnesses are given by the simple expressions $D_{0}^{x}=E_{0}^{x}=(J S / 2)(1-\eta)$ and $D_{0}^{y}=E_{0}^{y}=J S$. The SW stiffnesses in the CAF phase are derived by using Eqs.(2) and (3) to evaluate $d \theta_{a} / d B$ and $d \theta_{b} / d B$ at zero field and by using the SW frequencies [14] to perform a small $q$ expansion of $\omega_{\mathbf{q}}^{2}$. After a lengthy calculation, we obtain

$$
\begin{gathered}
D_{0}^{x}=\eta D_{0}^{y}=J S \sqrt{2} \eta \sqrt{1-\sqrt{\frac{\eta}{\eta+1}}}, \\
E_{0}^{x}=\eta E_{0}^{y}=\frac{J S}{2} \frac{3 \eta+(1-\eta) \sqrt{\eta /(\eta+1)}}{\sqrt{2+\sqrt{(\eta+1) / \eta}}} .
\end{gathered}
$$

For $\eta>1 / 3, E_{0}^{\alpha}>D_{0}^{\alpha}$ so that the SW stiffnesses are enhanced in the limit of small $q$ for fixed field. The ratio $E_{0}^{x} / D_{0}^{x}=E_{0}^{y} / D_{0}^{y}$ grows with increasing $\eta$. In the limit $\eta \rightarrow \infty$ as $\theta_{a} \rightarrow \pi / 6$ and $\theta_{b} \rightarrow \pi / 2, E_{0}^{\alpha} / D_{0}^{\alpha} \rightarrow \sqrt{\eta / 3}$. Also in the limit of large $\eta, D_{0}^{x} \rightarrow J S \sqrt{\eta}$ diverges but $D_{0}^{y} \rightarrow J S / \sqrt{\eta}$ tends to zero. 


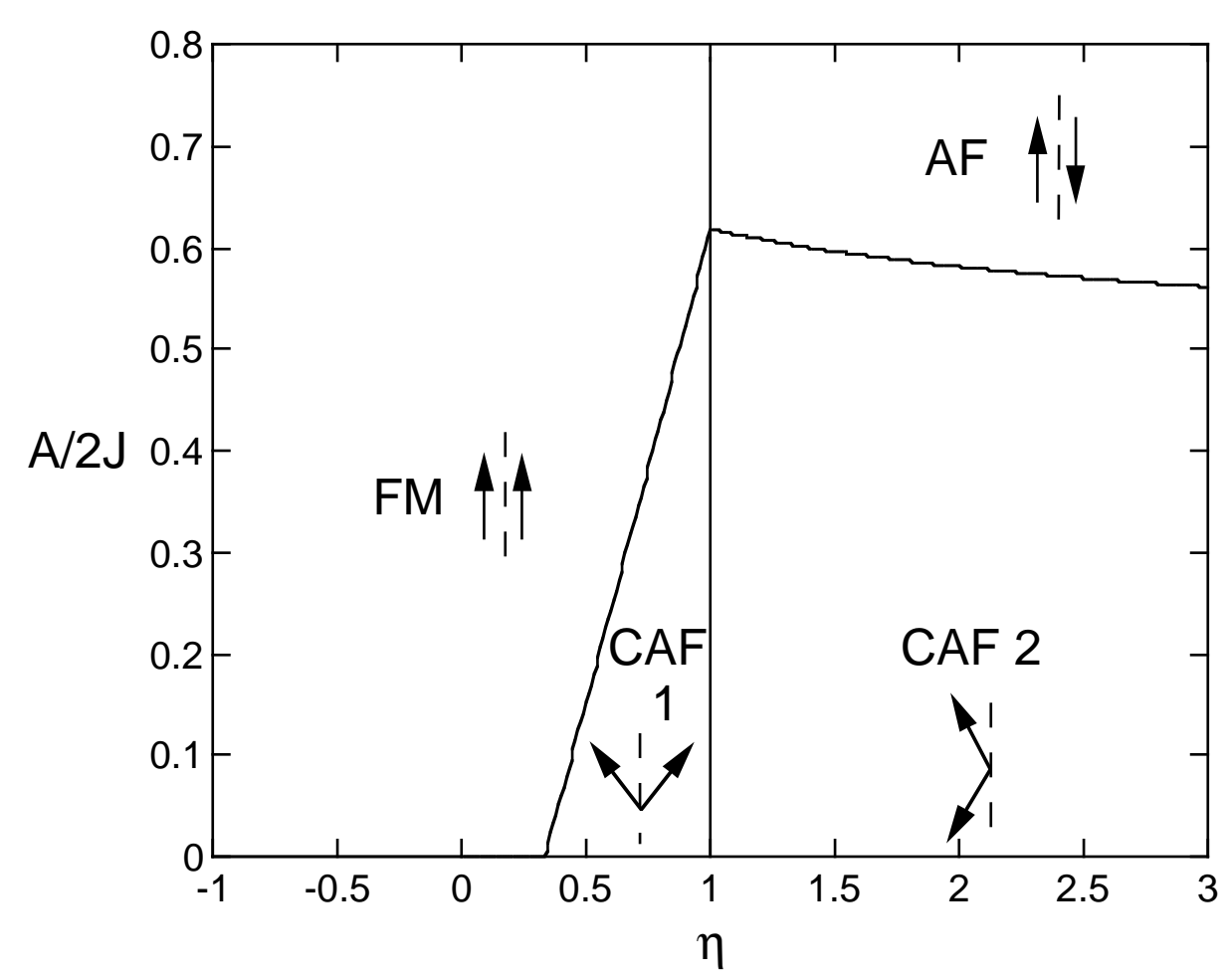

FIG. 2: The phase diagram of the GVA model with $B=0$. Two CAF phases differ in the orientation of the spins with respect to the anisotropy axis, which is drawn as the dashed vertical line.

The SW stiffness $D_{\mathrm{sw}}^{x}\left(q_{x}, B\right)=\left(\omega_{\mathbf{q}}-B\right) / q_{x}^{2}$ is plotted versus field in Fig.1 for $\eta=3$ and for several different values of $q_{x} / \pi$. In the limit $q_{x} \rightarrow 0$ for a small but fixed field, $D_{\mathrm{sw}}^{x} \rightarrow E_{0}^{x} \approx 2.05 J S$. But when $B \rightarrow 0$ at a small but fixed $q_{x}, D_{\mathrm{sw}}^{x} \rightarrow D_{0}^{x} \approx 1.55 J S$. In practice, neutron-scattering measurements in a FM or CAF must avoid the scattering from the lattice Bragg peak at $\mathbf{q}=0$ and the smallest wavevector used to measure the SW frequencies is about $0.08 \pi$. For this wavevector, higher-order corrections in $q^{2}$ contribute to Eq.(11) but the SW stiffness in Fig.1 still increases by roughly $15 \%$ as the field increases from 0 to $0.1 J S$. We emphasize that the dramatic increase in $D_{\mathrm{sw}}^{x}$ for small fields is not due to the changes in the equilibrium angles $\theta_{a}$ and $\theta_{b}$, which are minimal, but rather to the general inequivalence of $D_{0}^{x}$ and $E_{0}^{x}$ in a CAF. However, for very small canting angles ( $\eta$ just above $1 / 3),\left(E_{0}^{\alpha}-D_{0}^{\alpha}\right) / D_{0}^{\alpha} \approx 9 \theta_{a}^{4} / 8$ so that the difference between $D_{0}^{\alpha}$ and $E_{0}^{\alpha}$ is proportional to the fourth power of $\theta_{a}$ and may not be detectable if the canting angles are too small.

Many purported CAF's like the manganites $\mathrm{La}_{1-x} \mathrm{Sr}_{x} \mathrm{MnO}_{3}$ and $\mathrm{La}_{1-x} \mathrm{Ca}_{x} \mathrm{MnO}_{3}$ [8] with $0.05 \leq x \leq 0.125$ have anisotropy gaps between 0.2 and $0.5 \mathrm{meV}$. To determine the effects 
of anisotropy on the field dependence of the SW stiffness, we add the single-ion anisotropy energy $-A \sum_{i} S_{i z}^{2}$ along the $z$ axis to the Hamiltonian of the GV model. Minimizing the energy $E$ of this new "GVA" model in zero field, we obtain the phase diagram in Fig.2. There are now four possible phases: a FM phase for small $\eta$, an AF phase for strong anisotropy and $\eta>1$, and two CAF phases. For $\eta<1$, the spins in phase CAF 1 are sufficiently aligned that the net magnetization points along the anisotropy direction. For $\eta>1$, the non-colinearity of the spins is large enough that the anisotropy energy is minimized when the magnetization lies in the $x y$ plane. The transition between phases CAF 1 and CAF 2 is first order with discontinuous changes in $\theta_{a}$ and $\theta_{b}$. By contrast, the transition from CAF 2 to the AF phase is second order, as is the transition from CAF 1 to the FM phase. In a magnetic field applied along the $z$ axis, the spins of the CAF 2 phase will bend towards the $z$ axis with four inequivalent angles. While a magnetic field clearly favors the CAF 1 phase over the CAF 2 phase, the resulting phase diagram is rather complicated.

An anisotropy gap only appears in the CAF 1 phase. For the CAF 2 phase, the spins and magnetization are still free to rotate about the $z$ axis but rigid rotations about the direction of the magnetization carry a penalty: $\omega_{0}=0$ but $\omega_{\mathbf{Q}}>0$. This is reversed in the CAF 1 phase: $\omega_{0}>0$ but $\omega_{\mathbf{Q}}=0$. The harmonic Hamiltonian of the CAF 1 phase maintains the form of Eq. (44) but with revised coefficients. In the FM phase, the energy gap is given by $\omega_{0}=B+2 A S$ and the SW stiffnesses are unchanged.

A difficulty in treating systems with anisotropy is that both the SW stiffness and energy gap must be extracted from measurements. Assuming that two wavevectors $q_{1}$ and $q_{2}$ are used to fit the form $\omega_{\mathbf{q}}=\Delta_{0}+D_{\mathrm{sw}} q^{2}$, then the extrapolated gap $\Delta_{0}$ may differ from the true $q \rightarrow 0$ gap $\omega_{0}$, as shown in the inset to Fig.3. Motivated by measurements on $\mathrm{La}_{0.88} \mathrm{Sr}_{0.12} \mathrm{MnO}_{3}$ with an anisotropy gap of $0.5 \mathrm{meV}$ and a SW stiffness of $57.5 \mathrm{meV} \AA^{2}$ [] (the lattice constant is $3.8 \AA$ ), we use two sets of parameters to compare the field dependence of $\omega_{0}$ and $\Delta_{0}$ with the latter averaged over the $x$ and $y$ directions. The wavevectors $q_{1}=0.1 \pi$ and $q_{2}=0.2 \pi$ lie within the range of wavevectors used to experimentally extract the energy gap and SW stiffness. Both sets of parameters $\{A / 2 J=0.05, \eta=0.61\}$ and $\{A / 2 J=$ $0.1, \eta=0.96\}$ in Fig. 3 yield the same gap $\Delta_{0}=0.125 J S$, which gives $0.5 \mathrm{meV}$ for a realistic exchange constant of $J S=4 \mathrm{meV}$ [7]. For the larger value of $A / 2 J=0.1$, a higher value of $\eta$ with more canted spins $\left(\theta_{a}=19^{\circ}\right.$ and $\left.\theta_{b}=63^{\circ}\right)$ is required to produce the same $\Delta_{0}$ as the smaller value of $A / 2 J=0.05\left(\theta_{a}=16^{\circ}\right.$ and $\left.\theta_{b}=50^{\circ}\right)$. 


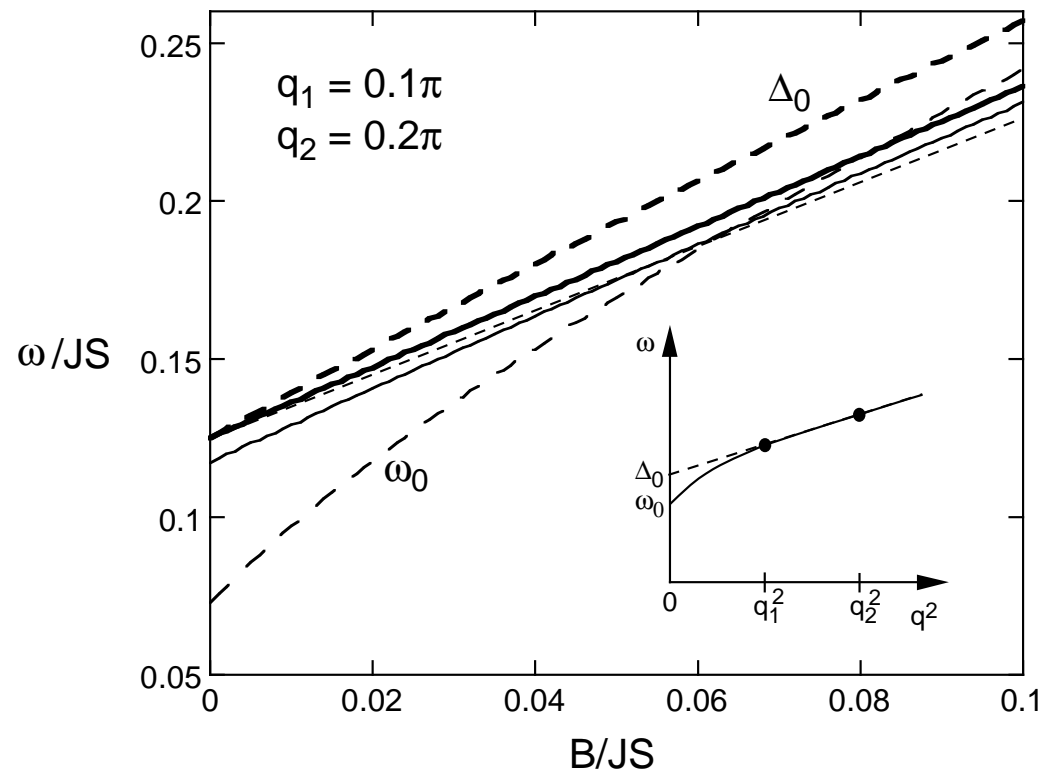

FIG. 3: The field dependence of the extrapolated gap $\Delta_{0}$ (thick curves) and the true $\mathbf{q}=0$ gap $\omega_{0}$ (light curves), using two values of $q / \pi$ as described in the inset and parameters $\{A / 2 J=0.05, \eta=$ $0.61\}$ (solid) and $\{A / 2 J=0.1, \eta=0.96\}$ (dashed). The small dash line is the field dependence $\Delta_{0}(B)=\Delta_{0}(0)+B$ required for a FM.

Because wavevectors between $q_{1}$ and $q_{2}$ fall into the moderate-to-high $q$ limit with $D_{\text {sw }} q^{2}$ comparable to or larger than the energy gap, we may estimate $\Delta_{0}$ by evaluating Eq.(II) in the large $q$ limit: $\omega_{\mathbf{q}} \approx\left(E_{0} / D_{0}\right) \omega_{0}+D_{0} q^{2}$, with an extrapolated gap of $\Delta_{0} \approx\left(E_{0} / D_{0}\right) \omega_{0}$. For the GVA model parameters in Fig.3, $\Delta_{0}$ overestimates $\omega_{0}$ by either $6.5(A / 2 J=0.05)$ or $71 \%(A / 2 J=0.1)$. Both $\Delta_{0}$ and $\omega_{0}$ increase with field as the difference between them diminishes. But as seen in Fig.3, the extrapolated gap $\Delta_{0}(B)$ increases more rapidly with field than it would for a FM. So if $\mathrm{La}_{0.88} \mathrm{Sr}_{0.12} \mathrm{MnO}_{3}$ is really canted, the difference (now reinstating $\left.2 \mu_{\mathrm{B}}\right) \Delta_{0}(B)-2 \mu_{\mathrm{B}} B$ should exhibit significant field dependence.

These results can also be applied to the SW measurements in the low-temperature phase of $\operatorname{Pr}_{1-x} \mathrm{Ca}_{x} \mathrm{MnO}_{3}$ with $0.3 \leq x \leq 0.4$. Originally believed to be a CAF [17, 18] both above and below the metal-insulator transition at $\sim 3.25 \mathrm{~T}$, this material is now thought by some to be phase separated [19, 20, 21]. Unlike the manganite discussed above, the anisotropy gap is negligible but $\Delta_{0}(B)$ may still be extrapolated from measurements in the large $q$ limit. 


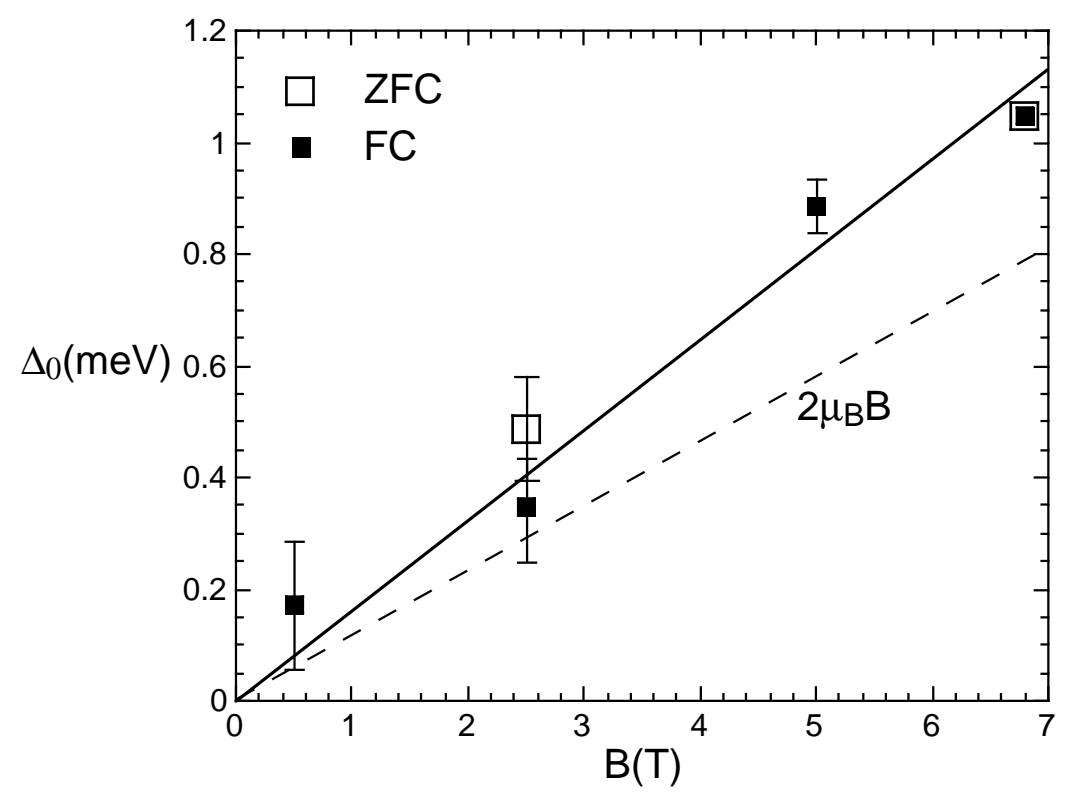

FIG. 4: The field dependence of the extrapolated gap for $\operatorname{Pr}_{0.7} \mathrm{Ca}_{0.3} \mathrm{MnO}_{3}$ with field cooled (FC) and zero-field cooled (ZFC) points shown 20]. The solid line is an interpolation through those points whereas the dashed line is the result $2 \mu_{\mathrm{B}} B$ required for a FM. For $6.8 \mathrm{~T}$, the error bars are smaller than the sizes of the FC and ZFC points.

For $x=0.3[20]$, the slope of the extrapolated gap $\Delta_{0}(B) \approx\left(E_{0} / D_{0}\right) \omega_{0}(B)$ plotted versus field in Fig. 4 is about $40 \%$ larger than the result $\omega_{0}(B) / B=2 \mu_{\mathrm{B}}$ required for a FM. While this discrepancy does not gainsay the evidence for phase separation in this compound [22], we conclude that the "FM" regions in $\mathrm{Pr}_{0.7} \mathrm{Ca}_{0.3} \mathrm{MnO}_{3}$ must be substantially canted with $E_{0} / D_{0} \approx 1.4$

Other canted systems should be amenable to a similar analysis of the extrapolated energy gap. Of particular interest are the CAF phases of the Dy and Ho intermetallics [6], which have large moments of over $6 \mu_{\mathrm{B}}$ and substantial canting angles. It would also be useful to perform this analysis on a wider range of FM materials. Perhaps because the result is self-evident, to our knowledge only a single FM material (MnSi above 0.62 T [23]) has been studied and shown to obey the required field dependence $\Delta_{0}(B)=\Delta_{0}(0)+2 \mu_{\mathrm{B}} B$.

To summarize, we have shown that the field dependence of the SW stiffness and extrapolated energy gap have unique signatures that distinguish a CAF from a phase-separated mixture containing FM and AF regions. Of course, magnetization measurements on single crystals [9] can also be used to identify CAF's. But considering the difficulty of those mea- 
surements, the field dependence of the extrapolated energy gap and SW stiffness provide important tools to identify and characterize CAF's. The results of this paper also have important implications for comparisons between the predictions of first-principles calculations and experiments, which may be describing behavior in different ranges of field and wavevector.

It is a pleasure to acknowledge helpful conversations with Drs. W. Saslow, M. Yethiraj, and A. Zheludev. I would especially like to thank J. Fernandez-Baca for sharing his unpublished data. This research was sponsored by the U.S. Department of Energy under contract DE-AC05-00OR22725 with Oak Ridge National Laboratory, managed by UT-Battelle, LLC.

[1] V.S. Khrapai, E.V. Deviatov, A.A. Shashkin, V.T. Dolgopolov, F. Hastreiter, A. Wixforth, K.L. Campman, and A.C. Gossard, Phys. Rev. Lett. 84, 725 (2000); D. Sánchez, L. Brey, and G. Platero, Phys. Rev. B 64, 235304 (2001).

[2] D. Coffey, T.M. Rice, and F.C. Zhang, Phys. Rev. B 44, 10112 (1991); L. Shekhtman, A. Aharony, and O. Entin-Wohlman, Phys. Rev. B 47, 174 (1993).

[3] J.D. Jorgensen, O. Chmaissem, H. Shaked, S. Short, P.W. Klamut, B. Dabrowski, and J.L. Tallon, Phys. Rev. B 63, 054440 (2001); G. Cao, S. McCall, Z.X. Zhou, C.S. Alexander, J.E. Crow, R.P. Guertin, and C.H. Mielke, Phys. Rev. B 63, 144427 (2001); K. Nakamura and A.J. Freeman, Phys. Rev. B 66, 140405(R) (2002);

[4] R. Welter, G. Venturini, E. Ressouche, and B. Malaman, J. Alloys Comp. 218, 204 (1995); G.J. Tomka, C. Ritter, P.C. Riedi, Cz. Kapusta, and W. Kocemba, Phys. Rev. B 58, 6330 (1998).

[5] J. van Duijn, J.P. Attfield, R. Watanuki, K. Suzuki, and R.K. Heenan, Phys. Rev. Lett. 90, 087201 (2003).

[6] C. Ritter, R. Cywinski, S.H. Kilcoyne, and S. Mondal, J. Phys.: Cond. Matt. 4, 1559 (1992); I. Mirebeau, I.N. Goncharenko, and I.V. Golosovsky, Phys. Rev. B 64, 140401(R) (2001).

[7] K. Hirota, N. Kaneko, A. Nishizawa, Y. Endoh, M.C. Martin, and G. Shirane, Physica B 237-238, 36 (1997).

[8] M. Hennion, F. Mousa, G. Biotteau, J. Rodríguez-Carvajal, L. Pinsard, and A. Revcolevschi, Phys. Rev. B 61, 9513 (2000); G. Biotteau, M. Hennion, F. Moussa, J. Rodríguez-Carvajal, L. 
Pinsard, A. Revcolevschi, Y.M Mukovskii, and D. Shulyatev, Phys. Rev. B 64, 104421 (2001); F. Moussa, M. Hennion, F. Wang, P. Kober, J. Rodríguez-Carvajal, P. Reutler, L. Pinsard, and A. Revcolevschi, Phys. Rev. B 67, 214430 (2003).

[9] J. Geck, B. Büchner, M. Hücker, R. Klingeler, and R. Gross, Phys. Rev. B 64144430 (2001).

[10] This rules out Dzyaloshinski-Moriya CAF's like the cuprates, which break inversion symmetry and have a linear SW dispersion, as shown for a simple one-dimensional model by Q. Xia and P.S. Riseborough, J. Appl. Phys. 67, 5478 (1990). The theory presented here still applies to such a system with the simple replacement of $q^{2}$ by $q$. But in the presence of a FM moment, a linear SW dispersion is a dead giveaway that the moments are canted and the unique signature of the SW velocity in a magnetic field may not be of quite as much interest.

[11] J. Villain, J. Phys. C 10, 1717 (1977).

[12] B. Berge, H.T. Diep, A. Ghazali, and P. Lallemand, Phys. Rev. B 34, 3177 (1986).

[13] M. Gabay, T. Garel, G.N. Parker, and W.M. Saslow, Phys. Rev. B 40, 264 (1989).

[14] R.S. Fishman, cond-mat/0404268

[15] L.R. Walker and R.E. Walstedt, Phys. Rev. B 22, 3816 (1980).

[16] W.M. Saslow and R. Erwin, Phys. Rev. B 45, 4759 (1992).

[17] Z. Jirak, S. Krupicka, Z. Simsa, M. Dlouha, and S. Vratislav, J. Magn. Magn. Mater. 53, 153 (1985).

[18] H. Yoshizawa, H. Kawano, Y. Tomioka, and Y. Tokura, Phys. Rev. B 52, R13145 (1995); J. Phys. Soc. Japan 65, 1043 (1996).

[19] I.G. Deac, J.F. Mitchell, and P. Schiffer, Phys. Rev. B 63, 172408 (2001).

[20] J.A. Fernandez-Baca, P.-C. Dai, H. Kawano-Furukawa, H. Yoshizawa, E.W. Plummer, S. Katano, Y. Tomioka, and Y. Tokura, Phys. Rev. B 66, 054434 (2002); (unpublished).

[21] Ch. Simon, S. Mercone, N. Guiblin, C. Martin, A. Brûlet, and G. André, Phys. Rev. Lett. 89, $207202(2002)$.

[22] The analysis of the extrapolated SW frequencies still holds for a phase-separated system so long as the smallest $q$ is larger than $2 \pi / L$, where $L$ is the size of the FM or CAF regions.

[23] J.A. Tarvin, G. Shirane, Y. Endoh, and Y. Ishikawa, Phys. Rev. B 18, 4815 (1978). 